Contributions to Game Theory and Management, XIV, 122-126

\title{
Cooperative n-person Prisoner's Dilemma on a Network*
}

\author{
Aleksandra L. Grinikh and Leon A. Petrosyan \\ St. Petersburg State University, \\ 7/9 Universitetskaya nab., Saint Petersburg 199034, Russia \\ E-mail: st062331@student.spbu.ru, 1.petrosyan@spbu.ru
}

\begin{abstract}
In the paper $n$-person prisoner's dilemma on the network is investigated. A cooperative game with the pairwise interaction of players is constructed. The model is a modification of the classic 2-person prisoner's dilemma problem in the game theory. Network interaction provide an ability to take into account the influence only to the adjacent players from the whole set of players. The feature of the game is found that allows to make a decision about necessity of playing dominated strategy by a few players. This solution is based on the number of the adjacent players. The work is a continuation of the paper published earlier by Grinikh A.L. and Petrosyan L.A. in 2021.
\end{abstract}

Keywords: pairwise interaction, cooperative game, prisoner's dilemma.

\section{Introduction}

Many game theory papers are devoted to two-person games. The classic example in this class of games is the two-person prisoner's dilemma game. The game represents a conflict of interests of two prisoners. Each of them is asked to reduce their prison sentences by extraditing their accomplice. Offers of betrayal are made to prisoners at the same time, and they have to decide to cooperate with the investigation immediately without being able to talk to their accomplices. Cooperation with the investigation reduces the term of imprisonment of the prisoner, even if his accomplice also decided to "give up", but extends the term of imprisonment of the accomplice.

In this paper we consider the $n$-person prisoner's dilemma that was proposed by Hamburger (1973), in his paper on N-person prisoner's dilemma. Straffin (1993) later developed his idea. He constructed a Nash equilibrium for a static game that was similar to the two-person model and shows the connections of the model to its prototype.

Here, this model is considered on the network. In this case, however, it is difficult to draw an analogy with the meaning that was the basis of the prisoner's dilemma. We think that this model can be used for any other dilemmas that arise in our lives and may have implications for the whole society. By looking at the model on a network, it is possible to model contemporary problems such as vaccination.

In such a problem statement, each of the players must decide to vaccinate himself or not (this corresponds to the strategies "to cooperate" / "to deviate" of the initial model). Vaccination is not an absolute guarantee not to get sick, but, when most of neighbors are vaccinated from their social networks, risk is almost minimal. That is why each of the players can increase the payoffs of all his relatives by

${ }^{*}$ This work was supported by the Russian Science Foundation under grant No.17-1101079 .

https://doi.org/10.21638/11701/spbu31.2021.11 
vaccinating, but since the vaccination itself can cause some discomfort, the dominant strategy remains "not to vaccinate" ("to deviate"). In this way, it is possible to define unequivocally that the prisoner's dilemma continues to be relevant, and moreover, its treatment in the case of $n$ players on the network adds the possibility of exploring a wider range of problems. In the case of a cooperative game, it will be possible to talk about finding the number of players whose vaccination will lead to the maximum gain of society (sufficient number of people will be brought into form "herd immunity").

\section{2. n-Person Prisoner's Dilemma on the Network}

\subsection{Model Description}

Let $\Gamma$ be a static game. A set of players is $N$ that has a cardinality $|N|=n$. All the players have two pure strategies: $C$ ("to cooperate") and $D$ ("to deviate"). Let the players be the nodes of a network $M$ that represents the connections between players as the edges of the network.

The payoff function of the player $i$ is $H_{i}$ that depends on the strategy of the player $i$ and the number of the adjacent players that choose the strategy $C, \forall i \in N$ :

$$
H_{i}\left(x_{1}, \ldots, x_{i}, \ldots, x_{n}\right)=\left\{\begin{array}{l}
C_{i}(x)=a_{1} x_{N}^{i}+b_{1}, \forall x \in(0, n], \text { if } x_{i}=C \text { and } x_{N}^{i} \\
\text { is the number of players from the set } N \text { that are } \\
\text { adjacent to the player } i \text { and choose } \\
\text { the strategy } C, \\
D_{i}(x)=a_{2} x_{N}^{i}+b_{2}, \forall x \in[0, n), \text { if } x_{i}=D \text { and } x_{N}^{i} \\
\text { is the number of players from the set } N \text { that are } \\
\text { adjacent to the player } i \text { and choose } \\
\text { the strategy } C .
\end{array}\right.
$$

Let $\bar{x}_{N}^{i}$ be the number of players from the set $N$ that are adjacent to the player $i$.

The payoff function $H_{i}\left(x_{1}, \ldots, x_{i}, \ldots, x_{n}\right)$ meets the following conditions:

(1) $a_{1} x_{N}^{i}+b_{1}<a_{2} x_{N}^{i}+b_{2}, \forall x_{N}^{i} \in\left[0, \bar{x}_{N}^{i}\right]$, since the strategy $D$ strictly dominates the strategy $C, \forall i \in N$.

(2) $a_{1} \bar{x}_{N}^{i}+b_{1}>b_{2}$, so, $H_{i}(C, \ldots, C)>H_{i}(D, \ldots, D)$.

(3) $a_{1} \geq a_{2}$ and $a_{1}>0, a_{2}>0$, therefore, $a_{1} x_{N}^{i}+b_{1} \geq b_{1}$ and $a_{2} x_{N}^{i}+b_{2} \geq b_{2}$.

\subsection{Cooperative Game}

Let $N=\{1,2, \ldots, n\}$ be the set of players. Any nonempty subset $S \subseteq N$ is called a coalition.

Definition 1. The cooperative solution is the profile of strategies that maximize the sum of all player's payoffs.

Definition 2. By a characteristic function of an $n$-person game we mean a function $V(S)$ that assigns a value to each subset of players $V: 2 N \rightarrow R$ in such a way, that $V(S)$ is the payoff to the subset $S \subseteq N$ if they maximize the sum of the payoffs of 
the players from the subset $S$, whereas the players from the set $N \backslash S$ act against $S . V(S)$ is called the value of the coalition $\mathrm{S}$ :

$$
V(S)=\max _{\mu_{S}} \min _{\nu_{N \backslash S}} H_{S}\left(\mu_{S}, \nu_{N \backslash S}\right), S \subseteq N,
$$

where $\mu_{S} \in \bar{X}_{S}, \nu_{N \backslash S}$ and $\bar{\Gamma}=\left(\bar{X}_{S}, \bar{X}_{N \backslash S}, H_{S}\right)$ is a mixed extansion of the zerosum game $\Gamma$.

The characteristic function represents the guaranteed total payoff for the players in a given coalition.

Theorem 1 (Cooperative solution). In the cooperative solution player $i$ will choose his pure strategy $C$, if the number of the adjacent players on the network $M$ satisfies the condition

$$
\bar{x}_{N}^{i} \geq \frac{b_{2}-b_{1}}{2 a_{1}-a_{2}} .
$$

Proof. Player $i$ should use the strategy $D$, if the gain of the player $i$ from choosing of the dominant strategy, in contrast to the strategy $C$ is greater than losses of the adjacent players from his "betraying".

Therefore, the player $i$ chooses the strategy $D$ to maximize the sum of payoffs of all the players from the coalition $N$, if the unequality $a_{1} \bar{x}_{i}^{N}<\left(a_{2}-a_{1}\right) \bar{x}_{i}^{N}+b_{2}-b_{1}$ holds.

Then, the player $i$ will choose the strategy $C$ in the cooperative solution, if

$$
a_{1} \bar{x}_{i}^{N} \geq\left(a_{2}-a_{1}\right) \bar{x}_{i}^{N}+b_{2}-b_{1} .
$$

Consequently, if $\frac{b_{2}-b_{1}}{2 a_{1}-a_{2}}$ is less than the number of players from the set $N$ that are adjacent to the player $i$, then this player will choose the strategy $C$ in the cooperative solution.

The same ratio can be found for the coalition $S \subseteq N$ :

If the number of players from the set $S \subseteq N$ that are adjacent to the player $i$ satisfies the relation $\bar{x}_{N}^{i} \geq \frac{b_{2}-b_{1}}{2 a_{1}-a_{2}}$, then in the cooperative solution the player $i$ will choose the strategy $C$. Otherwise, he will choose $D$.

Therefore, the characteristic function of a cooperative game for $n$-person prisoner's dilemma on the network can be written as:

$$
\begin{gathered}
V(i)=b_{2} ; \\
V(S)=\sum_{i \in S} H_{i}\left(x_{j}=C, \text { if } \bar{x}_{S}^{j} \geq \frac{b_{2}-b_{1}}{2 a_{1}-a_{2}} \text { and } j \in S, \text { otherwise } x_{j}=D\right) ; \\
V(N)=\sum_{i \in N} H_{i}\left(x_{j}=C, \text { if } \bar{x}_{N}^{j} \geq \frac{b_{2}-b_{1}}{2 a_{1}-a_{2}}, \text { otherwise } x_{j}=D\right) .
\end{gathered}
$$

In case, if it is possible to construct this characteristic function for our vaccination dilemma, it will be possible to find the number of people required to be vaccinated and, moreover, to know for what number of interactions, a person should give preference to vaccination in order to achieve herd immunity. 
Example 1 (3-person game).

Consider the payoff function for the 3-person prisoner's dilemma game on the network $M$ :

$$
H_{i}^{M}\left(x_{1}, x_{2}, x_{3}\right)=\left\{\begin{array}{l}
C_{i}(x)=16 x_{N}^{i}+1, \text { if } x^{i}=C ; \\
D_{i}(x)=16 x_{N}^{i}+18, \text { if } x^{i}=D .
\end{array}\right.
$$

The network $M$ for this game is represented in the figure 1 .

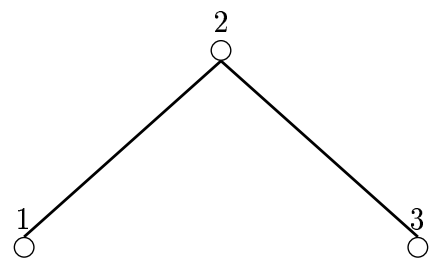

Fig. 1. An example of the network $M$ for the 3-person prisoner's dilemma game.

Next, we construct the characteristic function of the resulting game on the basis of ratios relative to the neighbors of each of the players. In accordance with the payoff function, player from the coalition $S$ should choose the strategy $C$, if the number of the adjacent to him players from this coalition is not less, than $\frac{b_{2}-b_{1}}{2 a_{1}-a_{2}}=$ $\frac{18-1}{2 * 16-16}=\frac{17}{16}$.

As we see from 1, this ratio can only be met for the second player if all players belong to the coalition $S$.

Table 1. This is the example table.

\begin{tabular}{ccc}
\hline$S$ & Unequalities & $V(S)$ \\
\hline$\varnothing$ & $1 \notin S, 2 \notin S, 3 \notin S$ & 0 \\
1 & $\bar{x}_{S}^{1}<\frac{18-1}{2 * 16-16}, 2 \notin S, 3 \notin S$ & 18 \\
2 & $1 \notin S, \bar{x}_{S}^{2}<\frac{18-1}{2+16-16}, 3 \notin S$ & 18 \\
3 & $1 \notin S, 2 \notin S, \bar{x}_{S}^{3}<\frac{18-1}{2 * 16-16}$ & 18 \\
$(1,2)$ & $\bar{x}_{S}^{1}<\frac{18-1}{2 * 16-16}, \bar{x}_{S}^{2}<\frac{18-1}{2 * 16-16}, 3 \notin S$ & 36 \\
$(1,3)$ & $\bar{x}_{S}^{1}<\frac{18-1}{2 * 16-16}, 2 \notin S, \bar{x}_{S}^{3}<\frac{18-1}{2 * 16-16}$ & 36 \\
$(2,3)$ & $1 \notin S, \bar{x}_{S}^{2}<\frac{18-1}{2 * 16-16}, \bar{x}_{S}^{3}<\frac{18-1}{2 * 16-16}$ & 36 \\
$N$ & $\bar{x}_{S}^{1}<\frac{18-1}{2 * 16-16}, \bar{x}_{S}^{2 * 16} \frac{18-1}{2 * 16-16}, \bar{x}_{S}^{3}<\frac{18-1}{2 * 16-16}$ & 69 \\
\hline
\end{tabular}

We can see that the maximum of the sum of players' payoffs for the grand coalition is not necessarily achieved by all players choosing a strategy $C$. This is not an obligatory condition for players' payoffs in the $n$-person prisoner's dilemma on the network game. Moreover, the lack of this assumption allows us to model situations such as vaccination. As we can see, we still have all the basic features of the game's prototype that is the two-person prisoner's dilemma.

\section{Conclusion}

In this paper, a cooperative game was constructed to model the $n$-person prisoner's dilemma on the network, with players having a pairwise influence on each 
other's payoffs. The proposed game model can address problems such as the number of vaccinated people that are needed to stop the pandemic. However, this study can be continued by considering not only the influence of adjacent players, but also the discounted influence of more distant players on the network.

\section{References}

Straffin, P. D. (1993). Game Theory and Strategy. The Mathematical Association of America: Washington, $244 \mathrm{p}$.

Hamburger, H. (1973). N-person prisoner's dilemma. Journal of Mathematical Sociology, 3, 27-48.

Grinikh, A. L., Petrosyan, L. A. (2021). An effective punishment for an n-person prisoner's dilemma on a network. Trudy Instituta Matematiki i Mekhaniki UrO RAN, 27(3), $256-262$. 